\title{
Analyzing the Mobility and Protocol Performance in MANET using a Novel Move Stop Deviate Model
}

\author{
$\mathrm{N}$ Vetrivelan \\ Research Scholar, NIT, Trichy, \\ Assistant Professor, Dept. of Computer Applications \\ Periyar Maniammai University, \\ Thanjavur-613403, India
}

\author{
Dr. A V Reddy \\ Professor, Dept. of Computer Applications \\ National Institute of Technology, \\ Trichy, India
}

\begin{abstract}
In this work, we have designed and implemented a novel Move Stop Deviate mobility model for Mobile ad hoc networks. In the MSD mobility Model, the node travels randomly for a total of 20 units before changing its direction, stops for a certain amount of time. Then it deviates angular direction (between angle $0,45,90,135,180,225,270,315$ only) and moves further. As soon as the boundary is reached, node gets back with the same velocity and traveling continuously at the same angle to reach the destination. The preceding process is repeated until the simulation termination condition is reached. The mobility metrics analysis has been done and also we have compared and analysed our novel MSD model with MANET protocols. The parameter metrics Packet Delivery Fraction, Routing load, and Latency have been taken into account. Our simulation result shows that the functioning of our MSD model has greatly influenced the performance of Routing Protocols in MANET environment. The result reveals the fact that the Reactive routing protocol DSR outperforms much more than the Proactive routing protocol DSDV. Our Novel Model has performed well when we compare it with existing Waypoint mobility model while setting many source-destination connections.
\end{abstract}

\section{Keywords}

Mobility, DSR, MANET, Move Stop Deviate and Simulation.

\section{INTRODUCTION}

In mobile ad hoc networks (MANET), a group of mobile nodes communicates with one another without a central control infrastructure. The network is vigorously changing and even they do not have any central administration system. The routes are multi hop due to available radio propagation range of wireless device. The network topology changes frequently due to random movement of nodes and thus prediction of network topology is very difficult. The trajectories of mobile nodes strongly influence MANET[5] performance. An Ad hoc routing protocols is a convention or standard that controls how nodes come to agree with a way to route packets between computing devices in a mobile ad hoc network, nodes do not have a prior knowledge of topology of network around them, they have to discover it. The basic idea is that a new node announces its presence and listens to broadcast announcements from its neighbors. The node learns about new near nodes and ways to reach them and announces that it can also reach those nodes.
The most common way to study mobile ad hoc networks is through simulations. Simulations[12] are fast and repeatable, and it is possible in simulators to isolate parameters affecting the performance of a design. A mobility model should attempt to mimic the movements of real MNs. Changes in speed and direction must occur and they must occur in reasonable time slots. We would not want MNs to travel in straight lines at constant speeds throughout the course of the entire simulation because real MNs would not travel in such a restricted manner. The Random Waypoint Mobility Model [1] is the 'bench mark' mobility model that is widely used in the current simulation environment. Nevertheless, RWMM [1] cannot accurately imitate all authentic mobility patterns in MANET. Therefore a variety of mobility models and communication pattern have been developed in the simulators for performance evaluation of a design. We show from our simulations results that MSD mobility Model has a considerable effect on the performance of these routing protocols [8] [9].

In Section 2 the related works on mobility modeling have been discussed. Brief description of MSD model has been presented in Section 3. Protocol description has been given in Section 4. In Section 5, the Simulation Models and Parameter Values have been described. Results and Discussion presented in Section 6. The conclusion has been presented in Section 7.

\section{RELATED WORKS ON MOBILITY MODELING}

Mobility model for simulations has been one of the important topics of research in this field. One of the early contributions was made by Broch et al., where they evaluated DSR, AODV, DSDV [8] and TORA using the Random Waypoint model [1]. They concluded that mobility has its impact on the performance of routing protocols. To evaluate these protocols over a wider range of scenarios, Johansson et al. proposed the scenariobased performance analysis. In this paper, they proposed mobility models for disaster relief, event coverage and conferences. Haas et al., introduces a mobility model in which the current velocity of a node may depend on. its previous velocity. Hong et al. proposed the RPGM model. One of the main applications of this model is in battlefield communications. The authors give several other applications of RPGM. While defining their framework they proposed to evaluate the protocols under a richer set of mobility models. Apart from using the RW and RPGM, they used two other 
mobility models, i.e., the FW and MH model. Tracy Camp, Jeff Boleng [1] surveyed the mobility models that are used in the simulations of Ad hoc networks. Authors described several mobility models that represent mobile nodes whose movements are independent of each other (i.e, entity mobility models) and several mobility models that represent mobile nodes whose movements are dependent on each other (i.e. group mobility models.) Per Johansson, Tony Larsson et al., compared three routing protocols for wireless mobile ad hoc network. They have done simulation on a scenario where nodes move randomly.

\section{THE WORKING PRINCIPLE OF A MOVE STOP DEVIATE MOBILITY MODEL}

There exist several mobility patterns that try to capture the behavior of the mobile devices under different circumstances. In this sense, MSD mobility model has been proposed. At the start of the simulation first creates the initial random position for the given number of nodes within the coverage area. In the MSD Model, the node travels randomly for a total of 20 units before changing its direction, stops for a certain amount of time. Then it deviates angular direction (between angle 0,45 , $90,135,180,225,270,315$ only) and moves further. As soon as the boundary is reached, node gets back with the same velocity and traveling continuously at the same angle to reach the destination. Here in this case the velocity of the nodes is independent. At the beginning of the each time interval, each node randomly chooses a speed between S_min and S_max. The preceding process is repeated until the simulation termination condition is reached.

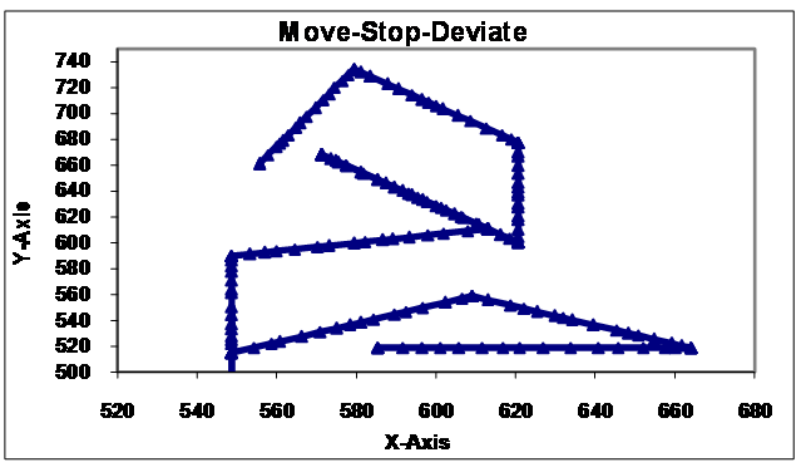

Figure 1 Movement Pattern of node under MSD Model

Figure 1 shows that the movement pattern of one particular node under MSD movement model with 100 nodes environment running with 100 Seconds. The nodes are moving, stops for a while and then moving random direction with given angles.

correlation, average node degree, total number of link changes and average link duration. we run the simulator of 100 nodes
The Destination $\mathrm{x}$ and $\mathrm{y}$ is calculated by

Destination_x $=$ Old_x + Random speed *

Cos(Angle*3.14/180)*interval

Destination $\_\mathrm{y}=$ Old $\_\mathrm{y}+$ Random speed $*$ Sin(Angle*3.14/180)*interval

\subsection{Parameters related to the model}

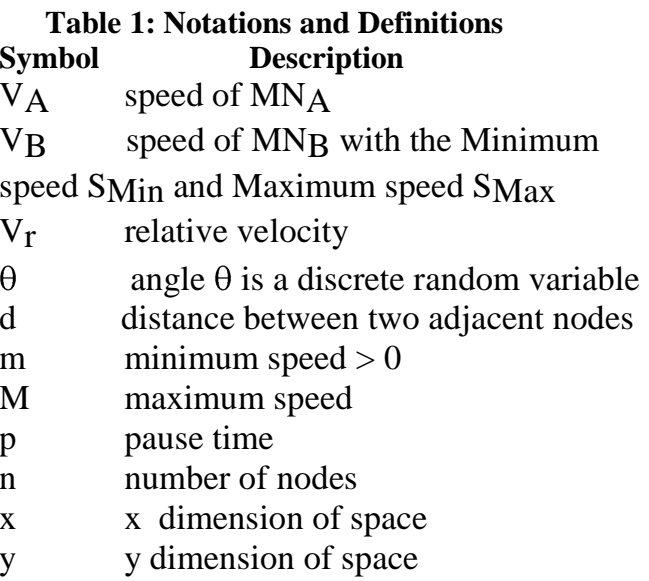

\subsection{Algorithm - MSD Model}

Algorithm 4.1: Procedure developed for the node movement for Restricted Angle Scenario Model

Step 1 : Load Start Time, Stop Time, Time interval, Speed Range and Angle. (Initial angle should be 0, 45, 90, 135, 180, $225,270,315$ degrees only)

Step 2 : Set initial node $\mathrm{x}$ and y position as a Random Location Step 3 : Pick a Random speed between Speed $\min \left(S_{\min }\right)$ and Speed max $\left(S_{\max }\right)$ value

Step 4 : Calculate next $x^{1}$ and $y^{1}$ from speed, time interval and angle of the node.

$$
\begin{aligned}
& \mathrm{x}^{1}=\mathrm{x}+\text { speed } * \cos (\text { angle }) * \text { interval } \\
& \mathrm{y}^{1}=\mathrm{y}+\text { speed } * \sin (\text { angle }) * \text { interval }
\end{aligned}
$$

Step 5 : If the new $\mathrm{x}^{1}, \mathrm{y}^{1}$ position touches the boundary, then it changes the movement to opposite direction.

Step 6 : If 20 Units completed, then change the angle to a random direction

Step 7 : After changing the angle then wait for few seconds

Step 8 : Repeat from step 3 until stop time reached.

\subsection{Mobility Performance Analysis}

To analysis the characteristics of the MSD mobility model, simulation experiments have been conducted for the mobility metrics such as relative speed, temporal correlation, spatial

network for 100 seconds with speed $0.5,1,1.5,2,2.5$ and $3 \mathrm{~m} / \mathrm{s}$ and topology dimension of $1000 \mathrm{~m} \times 1000 \mathrm{~m}$.

(i) Node Degree (ND) is defined as the number of neighbor nodes averaged over the amount of nodes and every time 
instant. The two nodes are neighbors if they are in transmission range $(t r)$. The node degree $N D$ is given by

$$
N D=\frac{\sum_{t=1}^{T} \sum_{\mathrm{I}=1}^{W} N\left(i_{i} t\right)}{T_{\mathrm{w}} N}
$$

(ii) Number of Link Changes : Number of link changes for a pair of nodes $i$ and $j$ is the number of times the link between them transitions from "down" to "up" during the simulation is given by

$$
\operatorname{LC}\left(\mathrm{i}_{0}, j\right)=\sum_{t=1}^{T} \mathbb{L C}\left(\mathbb{i}_{0} j_{0}, t\right)
$$

(iv) Link Duration: For two nodes i and j, at time t1,duration of the link (i,j) is the length of the longest time interval during

(iii) Degree of spatial dependence: It is a measure of the extent of similarity of the velocities of two nodes that are not too far apart.

$$
D_{\text {gpatial }}\left(\tilde{I}_{0} \tilde{j}_{0} t\right)=R D\left(\vec{V}_{0}(t) \vec{V}_{j}(t)\right) \in S R\left(\vec{V}_{0}(t) \vec{V}_{j}(t)\right)
$$

which the two nodes are within the transmission range of each other

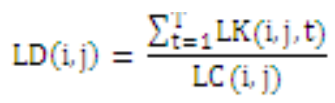

(v) Relative Speed : the standard definition drawn from physics, i.e. $\quad R s\left(\bar{D}_{0} j_{0} t\right)=\left\|\bar{V}_{i}(t)-\bar{V}_{j}\right\|$

(vi) Degree of temporal dependence: It is a measure of the extent of similarity of the velocities of a node at two time slots that are not too far apart. It is a function of the acceleration of the mobile node and the geographic restrictions.

$$
\begin{aligned}
& D_{\text {temporal }}\left(t_{w} t_{w} t^{0}\right)= \\
& D\left(\vec{V}_{0}(t) \vec{V}_{0}\left(t^{0}\right)\right)=S R\left(\vec{V}_{0}(t)_{0} \vec{V}_{0}\left(t^{0}\right)\right)
\end{aligned}
$$

In Table. 2 provides the analytical results of our MSD Mobility Model on various mobility metrics. Our mobility

\begin{tabular}{|c|c|c|c|c|c|c|}
\hline Speed & $0.5 \mathrm{~m} / \mathrm{s}$ & $1 \mathrm{~m} / \mathrm{s}$ & $1.5 \mathrm{~m} / \mathrm{s}$ & $2 \mathrm{~m} / \mathrm{s}$ & $2.5 \mathrm{~m} / \mathrm{s}$ & $3 \mathrm{~m} / \mathrm{s}$ \\
\hline Link Changes & 2031 & 2763 & 298018 & 444340 & 354566 & 297724 \\
\hline Link Duration & 33.40488 & 27.15658 & 1.302562 & 1.207527 & 1.758386 & 2.295434 \\
\hline Node Degree & 8.136238 & 8.620198 & 38.49446 & 53.71584 & 62.59089 & 67.77029 \\
\hline Relative Speed & 5.811198 & 5.327357 & 3.034719 & 1.956489 & 1.554732 & 1.339849 \\
\hline Spatial Correlation & 0.511084 & 0.550709 & 0.421134 & 0.360808 & 0.290449 & 0.238947 \\
\hline Temporal Correlation & 0.030674 & 0.037816 & 0.123833 & 0.179056 & 0.113672 & 0.077163 \\
\hline
\end{tabular}
pattern consisted of 100 mobile nodes moving in a simulation area of $1000 \mathrm{mx} 1000 \mathrm{~m}$ for the duration of 100 seconds. The velocity is varied from $0.5 \mathrm{~m} / \mathrm{s}, 1.0 \mathrm{~m} / \mathrm{s}, 1.5 \mathrm{~m} / \mathrm{s}, 2.0 \mathrm{~m} / \mathrm{s}, 2.5 \mathrm{~m} / \mathrm{s}$ and $3 \mathrm{~m} / \mathrm{s}$.

Table 2 Mobility Metrics of MSD Model

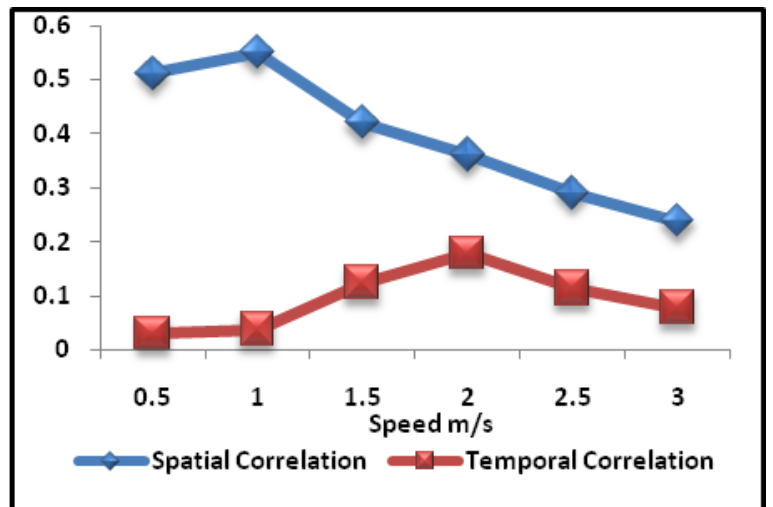

Figure 2 Spatial \& Temporal Correlation vs Speed

In Figure 2, the two plots spatial and temporal correlation on the graph are lower, this is due to that two nodes will not travel in the same direction or speed. This model has low temporal correlation because in each instant of time the motion of a mobile node in this mobility model is different (independent) to its motion in previous instance of time. In this model nodes move away to each other according to algorithm with different direction angle therefore this mobility model has very low degree of spatial correlation.

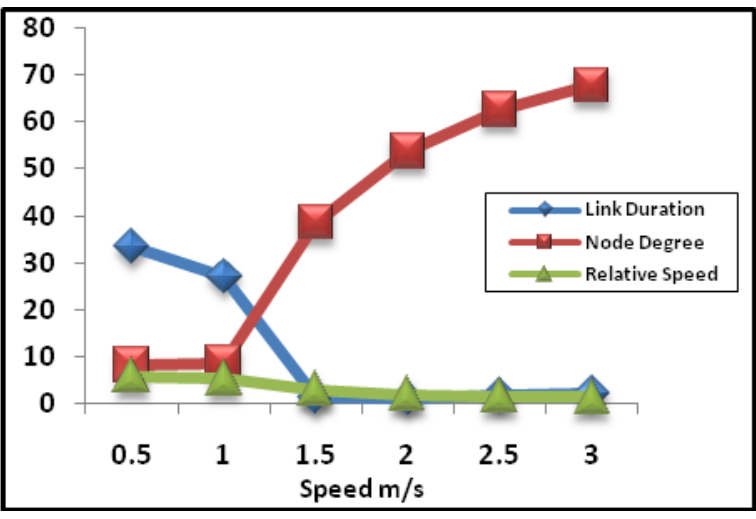

Figure 3 Link Duration, Node Degree \& Relative Speed

In Figure 3, the motion manner in the mobility model random selection of initial position, moving with restricted steps, the nodes stop on the regions of simulation area for a random time called pause time, then a direction change according to algorithm. The average relative speed is lower value while varying the speed of nodes in MSD mobility model. This is because of the nodes are moving different direction angle. As far as link changes are concern it is increasing trend. The link duration is high at lower speed of the movement of the mobile nodes, but duration is low at higher speed of the node. The status of a link between every pair of nodes within the transmission range of each other is monitored during the simulation. The link duration is calculated as the interval 
between the time when the link is created and time when it breaks. The node degree is increasing, while increasing the speed of the movement of the mobile nodes in the simulation grid.

\section{PROTOCOL DESCRIPTION 4.1 Dynamic Source Routing - DSR}

The key distinguishing feature of DSR [8] is the use of source routing. That is, the sender knows the complete hop-by-hop route to the destination. These routes are stored in a route cache. The data packets carry the source route in the packet header. When a node in the ad hoc network attempts to send a data packet to a destination for which it does not already know the route, it uses a route discovery process to dynamically determine such a route. Route discovery works by flooding the network with route request (RREQ) packets. Each node receiving a RREQ rebroadcasts it, unless it is the destination or it has a route to the destination in its route cache. Such a node replies to the RREQ with a route reply (RREP) packet that is routed back to the original source. RREQ and RREP packets are also source routed. The RREQ builds up the path traversed across the network. The RREP routes itself back to the source by traversing this path backward. The route carried back by the RREP packet is cached at the source for future use. If any link on a source route is broken, the source node is notified using a route error (RERR) packet.

\subsection{Destination Sequenced Distance Vector - DSDV}

DSDV [4] is a hop-by-hop distance vector routing protocol. It is proactive; each network node maintains a routing table that contains the next-hop for, and number of hops to, all reachable destinations. Periodical broadcasts of routing updates attempt to keep the routing table completely updated at all times. To guarantee loop-freedom DSDV uses a concept of sequence numbers to indicate the freshness of a route. A route $R$ is considered more favorable than $\mathrm{R}^{\prime}$ if $\mathrm{R}$ has a greater sequence number or, if the routes have the same sequence number, $R$ has lower hop-count. The sequence number for a route is set by the destination node and increased by one for every new originating route advertisement. When a node along a path detects a broken route to a destination $\mathrm{D}$, it advertises its route to $\mathrm{D}$ with an infinite hop-count and a sequence number is increased by one. Route loops can occur when incorrect routing information is present in the network after a change in the network topology, e.g., a broken link. In this context the use of sequence numbers adapts DSDV to a dynamic network topology such as in an ad-hoc network.

\subsection{Protocol Performance Metrics}

To evaluate the MSD mobility model in MANET, we used three performance metrics to compare and analyse the realistic movements.

4.3.1 Packet Delivery Fraction : The ratio of number of data packets successfully delivered to the destination, generated by CBR Sources. $\mathrm{PDF}=($ Received Packets/Sent Packets $) * 100$
4.3.2 Routing Overhead : It is an important metric for measuring scalability of a protocol. The number of routing packet transmitted per data packet delivered at destination. Each hop wise transmission of a routing packet is counted as one transmission. Routing load $=$ Packets sent $/$ Received packet 4.3.3 Latency: The time, it takes for a packet to cross a network connection from sender to receiver

\section{SIMULATION MODEL AND PARAMETERS}

In this section, the network simulation was implemented using the NS-2 simulation [12] tool. The Network Simulator NS-2 was a discrete event simulator. For simulation Scenario and network topology creation, it uses OTCL (Object Tool Command Language). To create new objects, protocols and routing algorithm or to modify them in NS-2, C++ source code used. The simulations were conducted on Due Core processor at speed $3.0 \mathrm{GHz}, 1 \mathrm{~GB}$ RAM running Linux Environment.

Table 3 Simulation Parameter Values

Parameter Value

$\begin{array}{ll}\text { Simulator } & \text { Ns2-2.34 } \\ \text { Number of Nodes } & 100 \\ \text { MAC Layer } & \text { IEEE } 802.11 \\ \text { Mobility Model } & \text { MSD Model } \\ \text { Topology x dimension } & 1000 \mathrm{~m} \\ \text { Topology y dimension } & 1000 \mathrm{~m} \\ \text { Transmission Range } & 250 \mathrm{~m} \\ \text { Antenna type } & \text { Omni directional } \\ \text { Minimum Speed } & 0 \mathrm{~m} / \mathrm{s} \\ \text { Maximum Speed } & 10 \mathrm{~m} / \mathrm{s} \\ \text { Pause time } & 5 \mathrm{~m} / \mathrm{s} \\ \text { Traffic Type } & \text { Constant Bit Rate } \\ \text { Packet size } & 512 \\ \text { Traffic rate (pkts/s) } & 10 \\ \text { Simulation Duration } & 100 \text { seconds } \\ \text { Source-Destination Traffic } & \text { udp }\end{array}$

\section{SIMULATION AND RESULTS}

We have analysed the performance of DSDV and DSR under MSD model in terms of Packet Delivery Fraction, Routing Load and Latency for varying source and destination traffic from 10,20,30,40 and 50 under 100 nodes environment.

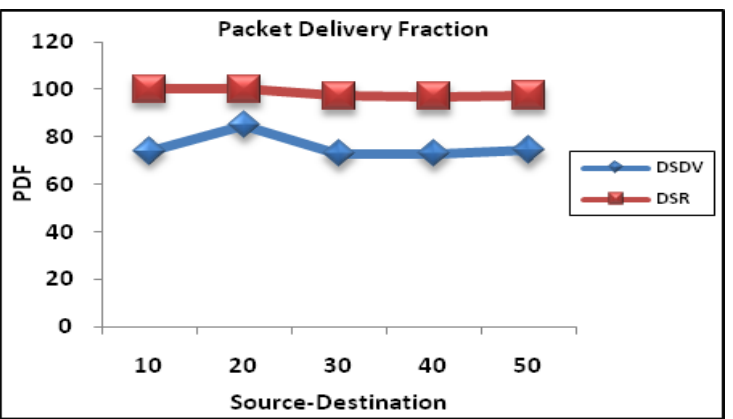

Figure 4 PDF - varying Source-Destination for routing protocols 


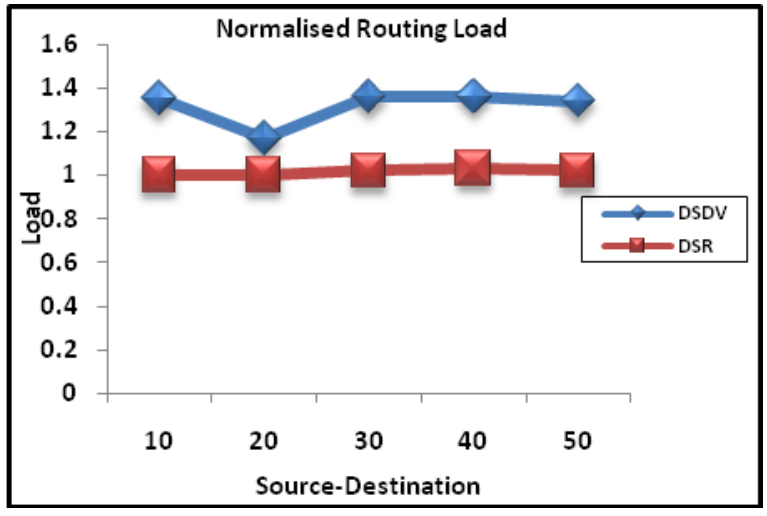

Figure 5 Routing Load - varying Source-Destination for routing protocols

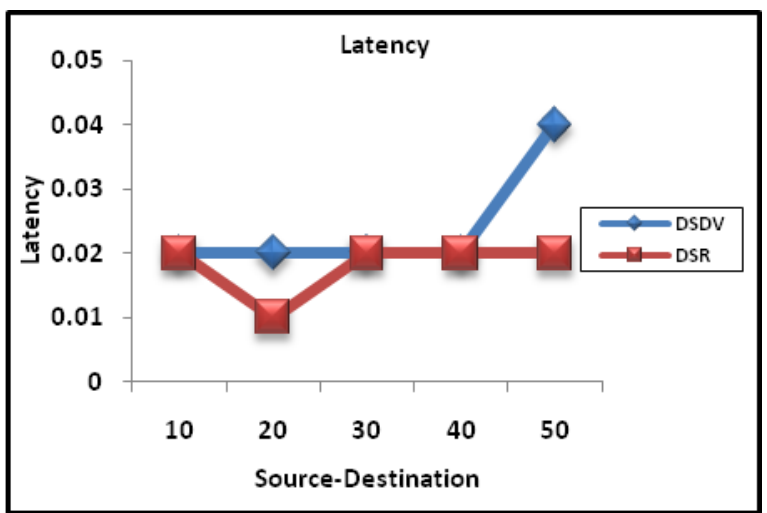

Figure 6 Latency - Varying Source-Destination for routing protocols

As shown in the Figure 4-6, we investigated the impact and effect of mobility on relative performance of protocols. As far as PDF, Routing Load and Latency are concerned DSR outperforms. Simulation experiments shown in Figure 4-6 confirm that for DSR under MSD, the PDF is highest between $97.07 \%-100 \%$, Routing Load is lowest between 1-1.030 and Latency is very less between $0.0144-0.0286$ seconds, in the case of DSDV under SSM, the PDF is $72.99 \%-85.06 \%$, Routing Load is $1.175-1.369$ and Latency is 0.0198-0.04551 seconds. We observed that DSR under MSD producing the highest performance. As far as PDF, Routing Load and Latency are concerned DSR outperforms. This is due to the networks with a dynamic topology, proactive protocols such as DSDV have considerable difficulties in maintaining valid routes, and loses many packets. It strives to continuously maintain routes to every node that increases network load as updations become larger. Route maintenance is much better in DSR as compared to DSDV. The reduction in performance can be attributed to link breakage, which is more probable as the length of the route increases. In case of DSDV re-establishment of new routes does not take place till there is a route table information packet coming from its neighbor nodes. But in case of DSR, when route breakage takes place, packets are cached and route repair takes place. This improves the overall performance of the system.

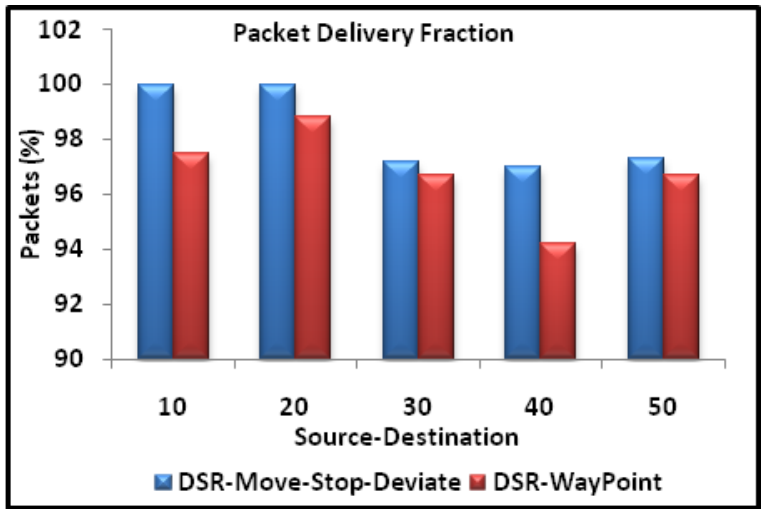

Figure 7 PDF- Comparison of MSD with Waypoint model

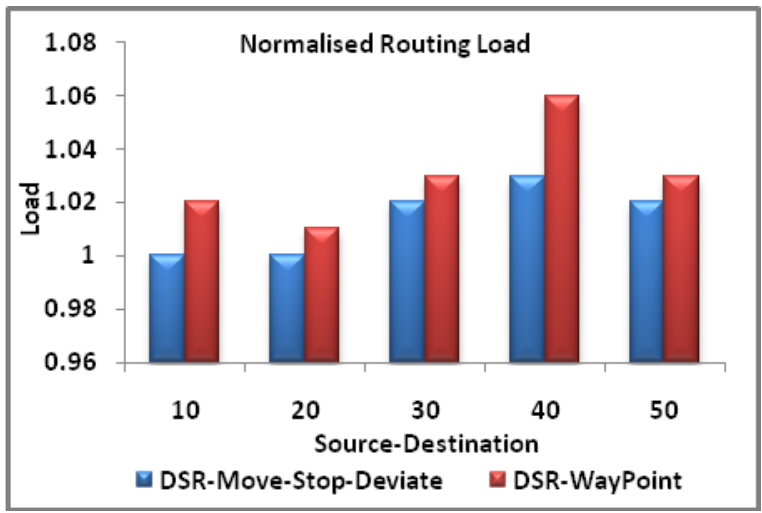

Figure 8 Routing Load- Comparison of MSD with Waypoint model

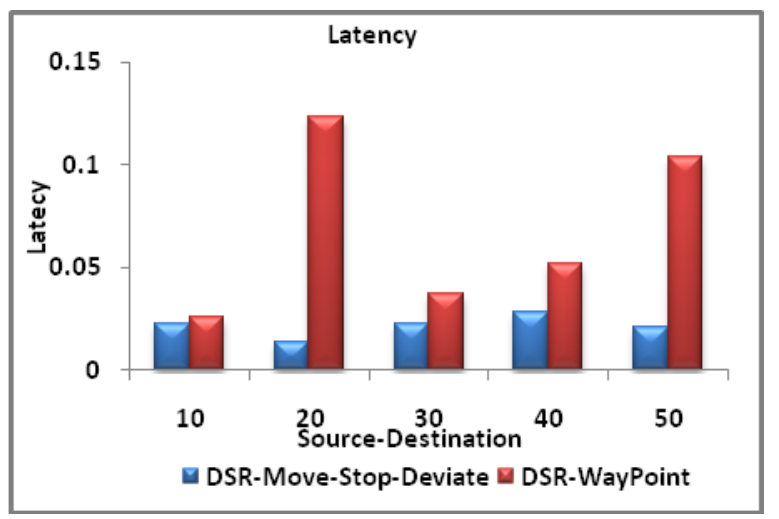

Figure 9 Latency- Comparison of MSD with Waypoint model

As shown in the Figure 7-9, we run the simulator for 100 seconds with 10, 20, 30, 40 and 50 udp connections (flows). The result reveals that our novel MSD Model performs better than the existing Way Point model for DSR protocol. From the results we can see that if compared the performance of MSD Model with Waypoint for higher number of SourceDestination. Simulation experiments shown in Figure 7-9 confirm that for DSR under MSD and Waypoint Model, PDF is between $93.56 \%-99.43 \%$ in MSD and in Waypoint $94.20 \%$ $98.88 \%$. Routing Load in MSD is 1.0056-1.068, Waypoint 1.01-1.06 seconds. Latency is between 0.016-0.049 in MSD and in Waypoint 0.026-0.1235 Seconds. MSD is comparatively performing better for DSR protocol. MSD Model is 
comparatively performing better for DSR protocol. The velocity of mobile nodes, which have memory less random process. i.e., Temporal Dependency. The mobile nodes are considered as an entity that moves independently of other nodes i.e., Spatial Dependency. The mobile node can move within simulation field with a restriction in accordance with the given angle. i.e., Geographic Restrictions of movement.

\section{CONCLUSION}

We designed, implemented and analysed the performance of the MSD mobility model with 100 nodes environment. The analytical results of our MSD Mobility Model on various mobility metrics have been done. The protocol performance metrics PDF, Routing Load and Latency have been taken to evaluate routing protocols DSR and DSDV. In our MSD Model, we have varied the Source-Destination flow vide 10,20,30,40 and 50. It has been found that DSR outperforms. The DSR discovers new route faster and more effectively to the destination when the old route is broken as it invokes route repair mechanism locally also high route cache hit ratio in DSR. Whereas in DSDV there is no route repair mechanism. In DSDV, if no route is found to the destination, the packets are dropped. While our novel MSD mobility Model is compared with the existing Waypoint model, the performance of MSD Model is better as far as PDF, Normalized Routing Load, and Latency are concerned. The reasons are the velocity of mobile nodes are memory less random process and they move independently over other nodes also the mobile node can move with a restriction in accordance with the given direction.

\section{REFERENCE}

[1] Azad, S., A. Rahman and F. Anwar, A performance comparison of proactive and reactive routing protocols of Mobile Ad-hoc Network (MANET). J. Eng. Applied Sci., vol.2(5), 2007 pp 891-896.doi : 10.1109/ICFN. 2009.64.

[2] Hong, X., Kwon, T.J., Gerla M., Gu, D.L and Pei, G., A Mobility Framework for Ad Hoc Wireless Networks, Lecture Notes in Computer Science, springer Berlin Vol. 1987, pp.185-196.doi: 10.1007/3.540-44498.

[3] Bai, F., G. Bhaskara and A. Helmy, Building the blocks of protocol design and analysis-challenges and lessons learned form case studies on mobile ad hoc routing and micro-mobility protocols. ACM Comput. Commun. 2004, vol.34 No.3, pp.57-70. doi: 10.1145/1031131.1031142.
[4] Johansson. P Larsson. T Hedman. N Scenario-based performance analysis of routing protocols for Mobile Adhoc Networks. Proc. Of ACM/IEEE International Conference on Mobile Computing and Networking, Seattle,Washington,USA,1999, pp.15-19.doi: $10.1145 / 313451.313535$

[5] Bettsltter, C., G. Resta and P. Santi, The node distribution of the random waypoint mobility model for wireless ad hoc networks. IEEE Trans. Mobile Comput., vol.2 issue 3,2003, pp.257-269,doi:10.1109/TMC.2003.1233531.

[6] C. de Waal, 2002 "Bonnmotion - a mobility scenario generation and analysis tool," www.cs.unibonn.de/IV/BonnMotion/.

[7] Camp, T., J. Boleng and V. Davies, A survey of mobility models for ad hoc network research. Wireless Communication and (WCMC): Special issue on mobile ad hoc networking. Res. Trends Appicat., Wiley and sons Publisher, 2002 vol.2: pp. 483-502.

[8] Corson, S. and J. Macker, Mobile Ad hoc Networking (MANET): Routing protocol performance issues and evaluation considerations. RFC, 2501, 1999.http://www.ietf.org/rfc/rfc2501.txt.

[9] Johnson, D.B. and D.A. Maltz, Dynamic Source Routing in Ad Hoc wireless networks. In:Mobile Computing, Imelinsky, T. and H.Korth (Eds.).,Chapter 5, Kluwer Academic

Publishers,Norwell,MA,USA.,ISBN:07792396979， 1996, pp:153-181.

[10] Lin, G., G. Noubir and R. Rajaraman, 2004. Mobility models for ad hoc network simulation. Proc. IEEE INFOCOM'04, 2004, pp.7-11.

[11] Mobility Generator (version http://nele.usc.edu/important/software.htm

[12] Network Simulator 2, http://www.isi.edu/nsnam/ns/

[13] Perkins, C.E. and E.M. Royer, Ad hoc on-demand distance vector routing. Internet-Draft, draft-ieft-aodv13.txt. $2003 \mathrm{http} / / /$ tools.ietf.org/id/draft-ietf-manet-aodv13.txt.

[14] Perkins, C.E. and P. Bhagwat, Highly dynamic Destination Sequenced Distance Vector routing (DSDV) for mobile computers. Proc. ACM SIGCOMM'94, Aug-Sep 1994. pp.234-244. doi : 10.1145/190314.190336 